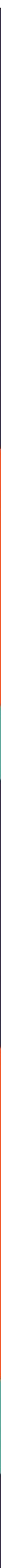

PARTICIPATION

\title{
Power to the patients
}

\section{When data-gathering precision-medicine projects build trust with their users, patients and researchers both benefit.}

\section{BY KATHERINE BOURZAC}

$\mathrm{I}$ 1996, Kathy Giusti was diagnosed with multiple myeloma, a rare and often fatal cancer. Her first bone-marrow biopsy took place on a Friday night. Outside the room, a group of scientists waited with an ice chest to take her bone-marrow sample. She told her doctor that she was surprised to see them working late. "He said my tissue was precious," she recalls.

For precision medicine to live up to its potential, millions of people must share their genomic data, their health records, and their experiences. To researchers, all of it is precious. The richer the databases, the better patient care will become.

This need gives ordinary people more power in medical research - not only to improve research quality by participating in greater numbers, but by speaking up and influencing what questions are asked in the first place. Despite lingering concerns over privacy (see page S70), it is patients and their loved ones who have been pushing for changes to the medical system that will enable personalized medicine, says Guisti, who founded the 
Multiple Myeloma Research Foundation with her sister in 1998.

For individual patients, empowerment takes a lot of time, work, education and economic resources. If programmes such as the United States' planned million-volunteer Precision Medicine Initiative Cohort Program are to succeed (see page S69), they must build people's trust and bring in not only highly motivated, seriously ill people, but healthy volunteers too. Early patient-centred research projects are already showing that this can pay off for researchers, for drug companies and, more importantly, for patients.

\section{ACCESS ALL AREAS}

It can be difficult for even well-educated, financially secure people living close to major US medical centres to access the best medical care. In 1998, Marty Tenenbaum was diagnosed with melanoma that had metastasized to his liver, "which in those days had zero survivors", he says. After a lot of searching, he was lucky enough not just to get onto a trial for an experimental therapy and surgery, but to be one of the few who responded. The trial failed, but Tenenbaum is still in remission.

This experience, and the genome-sequencing boom, led Tenenbaum - a computer scientist and former professor at Stanford University - to found a personalized-medicine consultancy called CollabRX in 2008. The company used bioinformatics to suggest therapies to its wealthy customers at a cost of US\$35,000-50,000. "My vision was to use information technology to close the loop between cancer research and clinical care," he says.

When CollabRX was acquired by Tegal in 2012, Tenenbaum wanted to start a non-profit organization that could serve more people. So he set up Cancer Commons, whose goal is to make the expertise of the best clinicians available to more cancer patients - especially those who cannot afford to travel to the best medical centres or pay for a personal consultation.

Tenenbaum sees buried treasure in the scientific discussions that take place behind closed doors on tumour boards - the groups of doctors, geneticists and researchers who discuss individual cases and decide on the best course of therapy. There are millions of possible combinations of drugs, many more than could ever be tested in clinical trials. Instead, the best oncologists and tumour boards are in effect experimenting on their patients, says Tenenbaum, trying new drug cocktails and seeing what happens. Yet little is learned: the deliberations and failed hypotheses of the tumour boards are not included in individual patients' records, and none of the data are shared outside the hospital. "There's all this experimentation and no learning," he says.

"Every patient presents a vastly complicated data set that we're barely able to interpret," says C. Anthony Blau, an oncologist at the University of Washington in Seattle who specializes in finding therapies for people with difficult-to-treat breast cancer. The ability to search a large pool of data on what has been tried with others would help oncologists to find the right treatment faster.

Tenenbaum is promoting this data-sharing vision through an online portal called Ask Cancer Commons. Patients or their caregivers can upload whatever medical records or genetic tests they have and give a description of their case. A group drawn from more than 100 volunteer oncologists and geneticists, including Blau, then reviews the case and gives feedback, serving as a virtual tumour board.

In the short term, Tenenbaum hopes that this will provide a lifeline for people who cannot access top-quality cancer care locally. But as more people take part, the database will grow. Patient data and the reports of the virtual tumour board are fed into a database that doctors can use to help future patients. Cancer Commons follows up to find out what the doctors did with their feedback, and how well it worked.

Hospitals are also contributing to the growth of the database. In a pilot test in 2015,50 cases from three tumour boards were summarized by volunteers and verified by doctors. Eventually, Cancer Commons will have enough of these hand-annotated data to construct algorithms that are capable of picking out important information from complex patient records and tumour-board deliberations without the need for volunteers at all. The project, known as the Insight Network, is currently fundraising and is expected to shift into full gear in the next few years.

As such projects grow, the need for people to take charge of their own care will diminish. "Avid patients will lead the way," says Blau. "The knowledge gained through them will be applicable to the population as a whole."

\section{MUTUAL BENEFIT}

One of the largest online data-sharing health projects, PatientsLikeMe, began in 2004 with a focus on neurological disorders. Since then, it has expanded to include 2,500 diseases. PatientsLikeMe now has about 500,000 users, most of them seriously ill. Through the website, these people can track their symptoms, join discussions and complete research surveys.

Jamie Heywood, a mechanical engineer and co-founder of the site PatientsLikeMe, calls it "a prospective epidemiology platform". So far, PatientsLikeMe has published more than 75 studies, mostly in collaboration with academic or corporate researchers. It has partnerships with the US Food and Drug Administration and drug company AstraZeneca among others, and it is financed by sharing patients' data with drug companies and researchers.

Heywood's younger brother, Stephen, was

diagnosed with amyotrophic lateral sclerosis (ALS) in 1998 at the age of 29, spurring Jamie to start the ALS Therapy Development Institute. It took the unusual approach of publishing results in real time as it screened drugs in mice and conducted a stem-cell trial with three participants, including Stephen.

People with a serious disease can feel very alone, says Hey-

"My vision was to use information technology to close the loop between cancer research and clinical care." wood. They want to be treated like partners, not subjects, and this is what PatientsLikeMe tries to do. Sharing data and experiences that may help others, and knowing that they are going to help researchers and drug companies, can make people feel heard and empowered (E. Chiauzzi et al. Patient http://doi.org/bpqw; 2016). "My brother died eight years ago and he's still helping people," says Heywood.

To build trust and encourage people to share data, the site is designed to be as accessible as possible. Instead of medical terms, patients use phrases such as 'brain freeze' to describe how they feel; the vernacular is then automatically matched with the standard medical code. The company also gets to know its users and adapts how it interacts with them accordingly. Some need to make a decision quickly after a diagnosis, for instance, whereas others with degenerative diseases have more time.

Unlike conventional top-down studies, in which data are collected on rigid timetables, PatientsLikeMe offers its users the flexibility to add data whenever they want, but this makes it less statistically rigorous than traditional research. "Real-world observational studies will never be able to match double-blind prospective studies in their ability to examine causality," says Heywood, and understanding biases in data contributed by users is a big challenge for epidemiologists and others at the company. But when patients are in charge, he says, they supply data that researchers do not normally have access to.

As an example of how patient-centred research can yield insights that benefit both patients and drug companies, Heywood points to a collaboration on insomnia between PatientsLikeMe, Northwestern University in Evanston, Illinois, and US drug company Merck \& Co. After developing a new sleep drug, surovexant, Merck approached PatientsLikeMe and asked it to look at the sleep patterns of members. Based on an initial survey of 75,000 users, Heywood says that his organization came up with about 50 hypotheses.

The team then narrowed both the set of questions and the study group. The resulting survey of just over 5,000 users in 2013 showed that only $13 \%$ had been diagnosed with insomnia, but that $73 \%$ of those who were 
undiagnosed also reported symptoms (B. Katic et al. Sleep Med. 16, 1332-1341; 2015). The data suggest that many people with serious illnesses have trouble sleeping - something that can exacerbate their condition, and doctors should be aware of the need to manage it. As the five-year study continues, participants will receive information about their individual sleep patterns.

Another of the site's self-tracking tools has been particularly useful for Allison Silensky, who has been using PatientsLikeMe since 2008. Silensky, who has a form of bipolar disorder, is a member of the company's user advisory board. The site's mood tracker has helped Silensky to notice and remember trends in her mood that she might otherwise have neglected to mention to her doctor. If she feels great at the doctor's office, she says, "I don't realize that the three weeks prior were horrible because I'm living in the moment."

At first her doctors warned her against getting involved with something on the Internet, she says, but they soon came to see the benefit. After a few years, Silensky saw a trend that she had not noticed before: all her hospitalizations were in spring. Now her doctor adjusts her medication in January, and her therapist checks on her more frequently in spring. She has not been hospitalized since.

\section{BUILDING TRUST}

People with serious and rare diseases, and their families, may be highly motivated to participate in data-sharing projects. But personalized medicine also needs healthy volunteers if researchers are to understand how diseases emerge. "This is a national movement, and we need everyone to participate," says Bray Patrick-Lake, director of patient engagement at the Duke Translational Medicine Institute in Durham, North Carolina.

Research and anecdote alike suggest that the possibility of helping others motivates people to share their data. Unpublished findings from a survey funded by the US National Institutes of Health suggest that proponents of precision medicine will have to convince people that they are contributing to the public good by sharing their data. Sandra Soo-Jin Lee, a biomedical ethicist, is looking at how diverse

"The biggest advocates for this are the patient groups, because they can see how it transforms health care."

communities feel about projects that link electronic health records with biobanks for research.

Her group at the Stanford Center for Biomedical Ethics in California is still analysing the results, which are based on surveys of 20 focus groups, including Hispanic, Asian and African American people, but already she has found "a tension" in people's attitudes. There is excitement that the data might lead to fresh targeted therapies or discoveries that are possible only with large pools of data. But some worry that the information will be used by the government for non-medical purposes, and others, says Lee, simply feel "a loss of control". And many are nervous about who will profit. “There's concern about who's actually going to use the data," she says - particularly that a third party will use the information to develop an expensive therapy.

Donating data and tissue for the public good is one thing, but often the benefits are not distributed equally. The HeLa line of immortal cancer cells, for instance, derived without

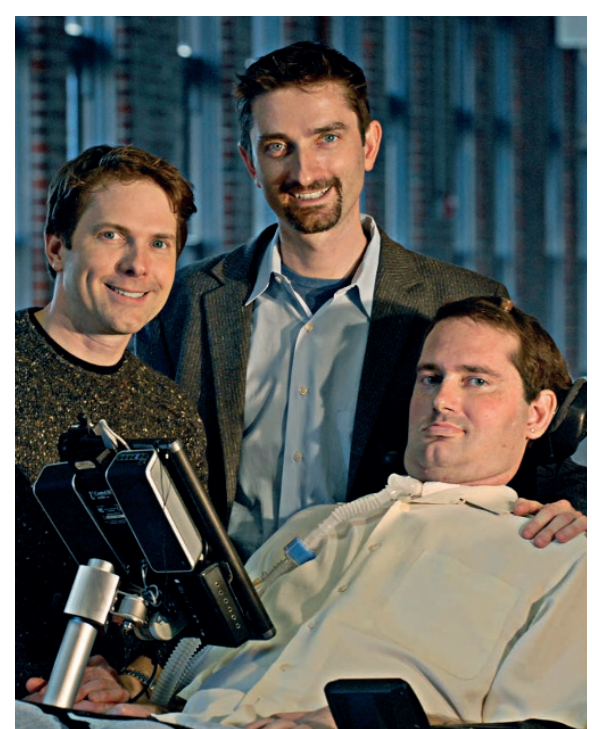

Jamie (left) and Ben Heywood (centre) set up

PatientsLikeMe when their brother Stephen was ill.

consent from the ovarian tumour of African American woman Henrietta Lacks, has long been a workhorse for cancer researchers. But the benefits of this research have not been distributed equally: cancer mortality rates for African Americans are still higher than those of any other ethnic group in the United States.

"In a fundamentally unequal health system, it's harder to argue that everyone should share," says Barbara Koenig, a medical anthropologist at the University of California, San Francisco. She studies the limits of informed consent in large public data-sharing projects. Without automatic enrolment for enterprises that serve the public good, most people will be motivated to opt into data sharing only when tragedy strikes in the form of a diagnosis for themselves or a loved one, says Koenig. "If people know they will benefit, they will share."

Heywood thinks that the people behind large government projects are misguided if they believe that "because they're trying to do the world good, the world will follow them". Building trust with people takes time, he says.

Continuing to carry out "onerous, top-down recruitment for clinical studies" is not working, says Sharon Terry, chief executive of the Genetic Alliance, a non-profit health advocacy organization. One of its projects, PEER, is an online resource that not only allows patients to make their information available to researchers, but also gives them control over how much of their health information is shared, and with whom, on a case-by-case basis.

"Our experiment is to use the tools of social media to engage people," says Terry. She thinks that the health-care industry does not do outreach as well as community organizations. So the Genetic Alliance is trying to learn from people in education and social services.

Today, precision-medicine portals, whether patient-driven or not, are fragmented, and that can make it hard to reach people, admits Giusti. But once people believe they are "on the path to a cure", they want to participate, she says.

\section{MAKING IT WORK}

The progress made by the Multiple Myeloma Research Foundation towards treating that particular cancer is due in part to the patients who willingly donated their tissue to a biobank in the hope of accelerating research. That project, called CoMMpass, was launched in 2011, and since then has validated several drug targets and treatment strategies. It has shown, for instance, that people treated with a combination of three drugs live longer without the disease progressing than those who are given only two. But these projects are expensive: CoMMpass cost more than $\$ 40$ million. "We struggle to see how this can be sustained," says Terry.

Large national, and ideally international, projects are the only way to make precision medicine work, says Kathryn North, leader of the Australian Genomics Health Alliance - and most people in the field accept that, she adds. Projects such as the US Precision Medicine Initiative are a great start, but they are only a start. There are economic, political and bureaucratic barriers to overcome, and probably only patients can make it happen. "The biggest advocates for this are the patient groups, because they can see how it transforms health care," says North.

The question facing patient advocates who want to see personalized medicine, says Terry, is this: "Can we impact the culture of large academic institutions, behemoth drug companies, and staid federal agencies?" Even if they want to change, there must be incentives driving them to do so. "I keep banking on public pressure, interest and rallying," she says. If precision medicine one day comes to benefit broad swathes of the population, it may well be thanks to a few patients who took it on themselves to push for that kind of future.

Katherine Bourzac is a freelance journalist based in San Francisco, California. 